\title{
Palmoplantar Erythrodysesthesia Associated with Capecitabine, Epidemiological Mapping and Risk Factors in Morocco
}

\author{
Tarik Hanafi", Hicham Titou, Hasna Kerrouch, Rachid Frikh, Naoufal Hjira and Mohammed Boui
}

Department of dermatology and venereology, Mohammed V Military Hospital, Faculty of Medicine and Pharmacy of Rabat, Mohammed V University, Morocco

*Corresponding author: Tarik Hanafi, Department of dermatology and venereology, Mohammed V Military Hospital, Faculty of Medicine and Pharmacy of Rabat, Mohammed V University, Hay Riad 10000 Rabat, Morocco

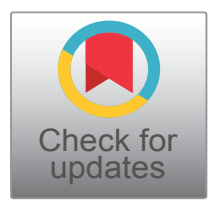

\begin{abstract}
A prospective cohort of a descriptive and analytical study, including sixty-one cases of Palmoplantar erythrodysesthesia in patients treated with capecitabine. The objective of this study is to determine an epidemiological mapping of Palmoplantar erythrodysesthesia in cancer patients on capecitabine treatment and to identify possible risk factors.

The incidence of PPE was $64 \%$. The median age of the patients who developed PPE was 54 years. $70 \%$ of the cohort was female, breast neoplasia represented $62 \%$ of all cases, $73 \%$ of these patients developed PPE. Colorectal neoplasia represented $27 \%$ of all cases, half of them developed PPE. $77 \%$ of our patients received a capecitabine monotherapy regimen, $59 \%$ of them developed PPE. $22 \%$ received a combined protocol including capecitabine, $81 \%$ of them developed PPE.

The risk factor identified in accordance with the data in the literature remains, combined treatment $(p=0.01)$. Risk factors identified in opposition to the data in the literature: sex $(p=0.04)$, primary breast neoplasia $(p=0.03)$. Comorbidities requiring new models of pathogenic apprehension and other more specific studies, diabetes $(p=0.03)$.
\end{abstract}

\section{Keywords}

Palmoplantar erythrodysesthesia, Capecitabine, Epidemiological mapping and Risk factors

\section{Introduction}

Palmoplantar erythrodysesthesia (PPE) or hand-foot syndrome or Burgdorf erythema [1] was first described in 1974 by Zuehlke [2], it is defined as a skin reaction commonly induced by a chemotherapeutic agent, char- acterized by mainly erythematous and painful palmoplantar involvement. 5-fluorouracil (5-FU), cytarabine, liposomal doxorubicin and capecitabine are the most frequently involved [3]. Capecitabine (Xeloda) is a fluoropyrimidine with antineoplastic activity. This agent is a systemic prodrug of 5-fluorouracil (5-FU), with the advantage of oral administration over the intravenous administration required with $5 \mathrm{FU}[4,5]$. The objective of this study is to determine an epidemiological mapping of EPP in cancer patients on capecitabine and to identify possible risk factors.

\section{Materials and Methods}

We ran a prospective, descriptive and analytical study from February 2016 to December 2019; we received all patients who are candidates for antitumor chemotherapy including capecitabine before the start of their treatment protocol. Inclusion criteria were, patients with solid malignant neoplasm whatever its metastatic status (metastatic or non-metastatic), and a chemotherapy protocol for curative, neoadjuvant or palliative antitumor chemotherapy based on capecitabine, whose protocol was 1250 $\mathrm{mg} / \mathrm{m}^{2}$ orally twice a day (morning and evening) for 2 weeks, followed by a week of rest, in 3-week cycles. Exclusion criteria were, Patients receiving or having received the following molecules: 5FU, Doxorubicin, Docitaxel and Cytarabine, and those with missing clinical information. Patients meeting the inclusion criteria received a first dermatological examination

Citation: Hanafi T, Titou H, Kerrouch H, Frikh R, Hjira N, et al. (2020) Palmoplantar Erythrodysesthesia Associated with Capecitabine, Epidemiological Mapping and Risk Factors in Morocco. Int J Cancer Clin Res 7:131. doi.org/10.23937/2378-3419/1410131

Accepted: January 25, 2020; Published: January 27, 2020

Copyright: (C) 2020 Hanafi T, et al. This is an open-access article distributed under the terms of the Creative Commons Attribution License, which permits unrestricted use, distribution, and reproduction in any medium, provided the original author and source are credited. 


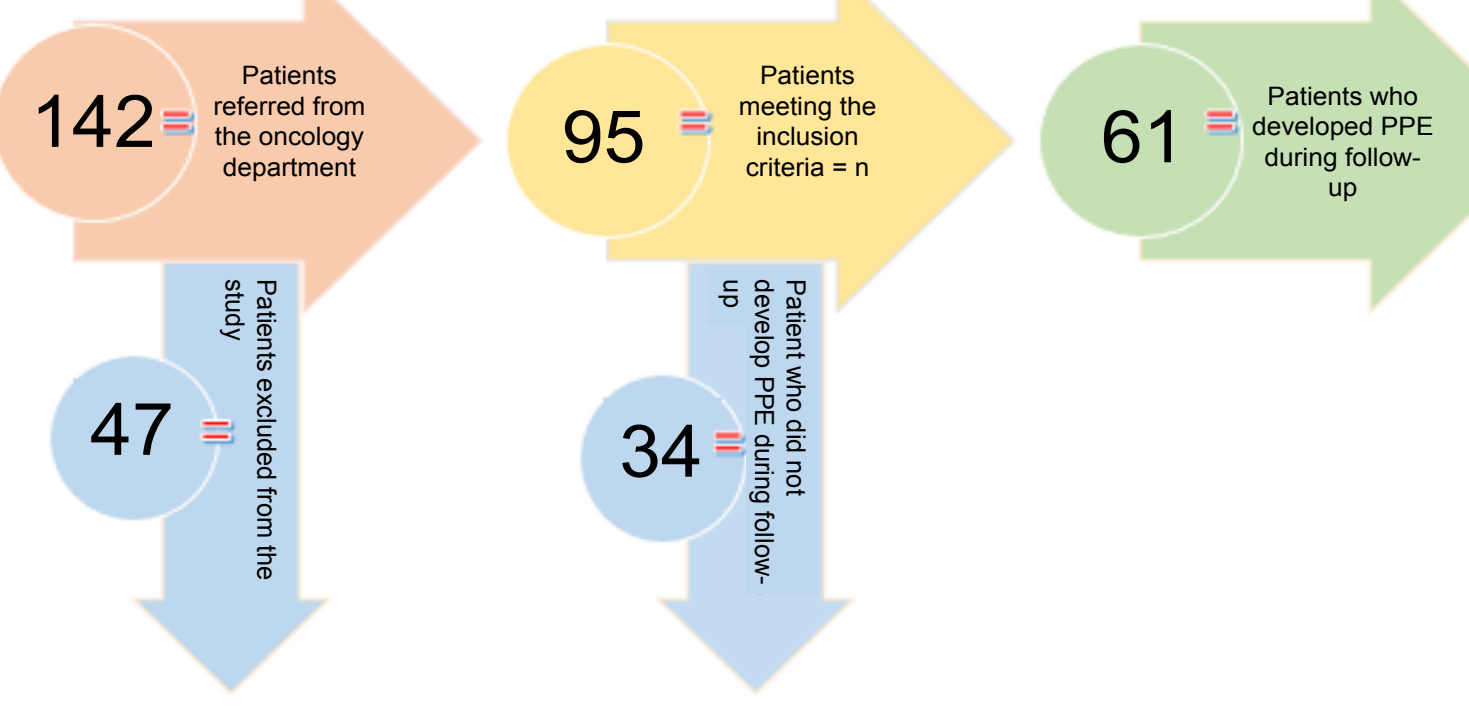

Figure 1: Study flowchart.

Table 1: Distribution of the study cases according to age, sex, site of primary neoplasia, monotherapy vs. combined treatment regimen.

\begin{tabular}{|c|c|c|c|}
\hline & $\begin{array}{l}\text { Total } \\
\mathrm{N}=95\end{array}$ & $\begin{array}{l}\text { Patients with PPE } \\
N=61\end{array}$ & $\begin{array}{l}\text { Patients without PPE } \\
N=34\end{array}$ \\
\hline Age in years. median \pm standard deviation & $52 \pm 10$ & $54 \pm 10$ & $50 \pm 09$ \\
\hline \multicolumn{4}{|l|}{ Sex } \\
\hline Man & $29(30)$ & $16(26)$ & $13(38)$ \\
\hline Woman & $66(70)$ & $45(73)$ & $21(61)$ \\
\hline \multicolumn{4}{|l|}{ Neoplasia. n (\%) } \\
\hline Mammary & $59(62)$ & $43(70)$ & $16(47)$ \\
\hline Colorectal & $26(27)$ & $13(21)$ & $13(38)$ \\
\hline Others & $10(10)$ & $5(8)$ & $5(14)$ \\
\hline \multicolumn{4}{|l|}{ Treatment } \\
\hline Combined & $21(22)$ & $17(27)$ & $4(11)$ \\
\hline Monotherapy & $74(77)$ & $44(72)$ & $30(88)$ \\
\hline
\end{tabular}

before the initiation of the first cycle of chemotherapy, with the characterization of epidemiological data including: Age, sex, primitive cancer site and the mode of chemotherapy (combined vs. monotherapy). We systematically looked for four pre-existing factors (History and/or progressive pathology): Eczema, dermatophytosis and/or onychomycosis, chronic venous insufficiency and diabetes. In follow-up, the diagnosis of PPE was retained on concordant clinical and chronological description based on the NCl-CTC (National Cancer Institute - Common Toxicity Criteria) Grading HFS (Hand-foot syndrome), the histological confirmation was not required. Statistical analysis was performed by Statistical Package for Social Sciences (SPSS) version 18.0. Univariate analysis was conducted using standard $v^{2}$ test or Fisher's test when it was not applicable. A value of $P$ less than 0.05 was considered significant. Multivariate analysis was performed by logistic regression after including all variables with a $P$ value $<0.20$.

\section{Results}

\section{Architecture of the study}

A total of one hundred and forty-two patients (patients who are candidates for antitumor chemotherapy including capecitabine) were referred from the medical oncology department to the dermatology consultation. Forty-seven of them were excluded from this study, mainly for two causes, the most important was because they already received or are going to receive one of the following molecules: 5FU, Doxorubicin, Docitaxel and Cytarabine, the second reason was because we will initiate a different capecitabine protocol. Thus, only twenty-five and fifteen patients were included in the study, that we divided into two groups in the follow-up after the start of chemotherapy, patients who have developed PEE: Sixty-one cases, patients who did not developed PPE: Thirty-four cases (Figure 1). 
Table 2: Distribution of the co-morbidities studied in our cohort.

\begin{tabular}{|l|l|l|l|}
\hline & Total & Patients with PPE & Patients without PPE \\
$\mathbf{N = 9 5}$ & $\mathbf{N = 6 1}$ & $\mathbf{N 4}$ \\
\hline Eczema & $12(12)$ & $08(13)$ & $04(11)$ \\
\hline Dermatophytosis and/or onychomycosis & $33(34)$ & $25(40)$ & $08(23)$ \\
\hline Chronic venous insufficiency & $16(16)$ & $10(16)$ & $06(17)$ \\
\hline Diabetes & $40(42)$ & $32(52)$ & $08(23)$ \\
\hline Association of two or more factors & $25(26)$ & $19(31)$ & $06(17)$ \\
\hline
\end{tabular}

Table 3: Statistical analysis of risk factors.

\begin{tabular}{|l|l|l|l|}
\hline & HR & $95 \%$ CI & Valeur $\mathbf{p}$ \\
\hline Age & 0.5 & $0.33-1.37$ & 0.40 \\
\hline Sex & 1.24 & $0.95-3.1$ & 0.04 \\
\hline Breast cancer & 1.40 & $0.76-2.58$ & 0.03 \\
\hline Colorectal cancer & 1.34 & $0.15-10.8$ & 0.8 \\
\hline Monotherapy & 0.4 & $0.33-21.2$ & 0.3 \\
\hline Combined treatment & 2.72 & $1.33-5.5$ & 0.01 \\
\hline Eczéma & 0.97 & $0.59-1.64$ & 0.94 \\
\hline Dermatophytosis and/or onychomycosis & 0.7 & $0.45-1.49$ & 0.49 \\
\hline Chronic venous insufficiency & 2.8 & $0.43-10.5$ & 0.35 \\
\hline Diabetes & 0.4 & $0.21-0.80$ & 0.03 \\
\hline Association of two or more factors & 0.51 & $0.29-0.98$ & 0.02 \\
\hline
\end{tabular}

\section{Epidemiological characterization}

Incidence: The number of patients who developed PPE was 61 out of 95 patients, corresponding to an incidence of $64 \%$.

Age: The age varied from 18 years to 75 years with a median of 52 years, the median age of the patients who presented with PPE was 54 years, slightly higher in comparison with the patients who did not developed PPE (50-years-old).

Gender: $70 \%$ of the cohort was female, $68 \%$ developed PPE versus $55 \%$ of men, and the sex ratio $\mathrm{M} / \mathrm{W}$ of the group with PPE was 0.35

Primitive site of neoplasia: Breast neoplasia represented $62 \%$ of all cases, $73 \%$ of these patients developed PPE, representing $70 \%$ of the total number of patients with PPE. Colorectal neoplasia represented $27 \%$ of all cases, $50 \%$ of these patients developed PPE, representing $21 \%$ of the total number of patients with PPE. For the other locations, i.e. $10 \%$ of all cases, half (5 patients) developed PPE, representing $8 \%$ of all patients with PPE.

Chemotherapy regimen: Combined vs. monotherapy: $77 \%$ of our patients received a capecitabine monotherapy regimen, $59 \%$ of them developed PPE. $22 \%$ of our patients received a combined protocol including capecitabine, $81 \%$ of them developed PPE Table 1.

\section{Comorbidities}

Eczema: $12 \%$ of our patients had a history and/or ac- tive eczema, $66 \%$ of whom developed PPE.

Dermatophytosis and/or onychomycosis: $34 \%$ of patients had dermatophytosis and/or onychomycosis, $75 \%$ of whom had PPE.

Chronic venous insufficiency: $16 \%$ of patients had a CVI, $62 \%$ of whom had progressed to PPE.

Diabetes: $42 \%$ of our patients had diabetes, of which $80 \%$ progressed to EPP.

Association of two or more factors: Was found in $26 \%$ of patients, $76 \%$ of whom developed PPE Table 2.

\section{Risk factors}

Demographically, the female sex seems to be correlated with a significant risk with $p=0.04$, which is not the case for age $(p=0.4)$. Primarily breast neoplasia $(p=0.03)$ and combined treatment $(p=0.01)$ compared to monotherapy ( $p=0.3$ ) seem to favour PPE. Among comorbidities, the results define diabetes as a statistically significant risk factor $(p=0.03)$, as well as the combination of two or more factors $(p=0.02)$. However, multivariate analysis found no independent risk factor Table 3.

\section{Discussion}

\section{Incidence}

The reported incidence of PPE in patients on capecitabine varies from $33 \%$ [6] to $68.3 \%$ [7], most authors report an incidence varying between $50 \%$ and $60 \%$ (all cancers combined) $[4,8]$, rare are the series with a lower rate, in particular that of gomez-martin, et al. [9] which 
reports an incidence of $19.6 \%$ (metastatic gastric cancer), justifying this rate by better use of capecitabine and optimized management of PPE, however in our series, almost two thirds (64\%) of our patients were complicated by PPE, this with the standardized methods and posology of use of chemotherapy protocols, $45 \%$ of them developed PPE during the first cycle, 32\% during the second, $16 \%$ during the third one and only $5 \%$ later. Indeed we believe that the prior information of patients regarding the dermatological toxicities of capecitabine and especially the availability and easy access to dermatological control has made it possible to recruit a significant number, this is particularly valid, for light and beginners shapes, who could not drive into consultation or have recourse to phytotherapy, the typical example in our context remains henna [10]. On the other hand, our patients have not received preventive treatment, in particular corticosteroids. The role of patient education [11] would be crucial in self-identification or even management of EPP earlier, to avoid progression at advanced stages requiring reduction or discontinuation of capecitabine.

\section{Age}

The median age in the various series in the literature varied between 48 years [12] and 69 years [13], extreme medians of 76 years [6] are reported in certain studies which have been exclusively interested in cancer patients of old age, who concluded in a very good tolerance with a very low incidence at $28 \%$, however, all the patients in this study were on monotherapy, which would partly explain the low incidence rate. The age of the patients in our study varied from 18 years to 75 years with a median of 52 years, the median age of patients with PPE was slightly higher (54 ans), our median is one of the lowest in the literature, largely explained by the prevalence and epidemiological profile of breast neoplasia in our country. In our series, age was not significant as a risk factor $(p=0.4)$, this agrees with the literature, in particular the work of Abushullaih, et al. However, Heo, et al. had determined that age $>52$ years was significant with $p=0.024$, however these results were associated with a regimen in combined treatment.

\section{Gender}

The female predominance $(\mathrm{M} / \mathrm{W}=0.35)$ results from an inhomogeneous sampling, with breast cancers which represents $62 \%$ of the cases of the study. If the female sex is recognized as a risk factor for PPE on 5 FU [14], it is not the same for capecitabine, Abushullaih, et al. [7], on a series of 41 patients (Metastatic colorectal cancers) did not find a significant risk related to sex. In another series [15] of 179 patients including 85 women, there was also no significant risk ( $p=0.151$ ). Suzuki, et al. [16] found an insignificant male predominance with 54 males out of 90 patients. On the contrary, our series finds a significant risk ( $p$ $=0.04$ ) linked to the female sex, particularly linked first to the predominance of breast neoplasia $(p=$ 0.03 ) but also to the lifestyle of our Maghreb sample, where housewives are more prone to housework involving overexposure to microtrauma and also to sudden changes in temperature.

\section{Initial site of neoplasia}

The incidence in colorectal cancer cases was 50\%, which agrees with data from the literature. In the study by hoff, et al. [17], of 302 cases of colorectal cancer treated with capecitabine, the incidence of PPE was $58 \%$. Van custem, et al. [18] reported an incidence rate of $48 \%$ in 301 cases.

In our series, the incidence of PPE in breast cancer cases was $72 \%$, which is largely high compared to the data in the literature. Reichardt, et al. [19], in a series of 136 cases validated an incidence of $55 \%$. Miller, et al. [20] reported an incidence of $60 \%$.

In our series, the calculation of the risk associated with breast localization was significant $(p=0.03)$ unlike the results of other studies [7], which had not found any risk in a small series of 41 patients. Heo, et al. [15], this time in a larger series of 179 patients, who, by opposing the breast, gastric and biliary localizations, did not find any significant risk correlated with the primary localization.

These results could also be linked to the reasons explained above in relation to the particular profile of women in the Maghreb, but this remains an impression which will require objectification and evaluation by other studies determining the psycho-social profile of cases of study.

\section{Chemotherapy regimen}

The $4 / 5$ of our patients receiving a combined treatment developed a PPE with a $(p=0.01)$, this rate decreases to $3 / 5$ in monotherapy $(p=0.3)$, in fact the combined treatment was objectified by Heo, et al. [15] as a risk factor, particularly in the association with docetaxel $(p=0.000)$, but also with cisplatin ( $p$ $=0.021)$ and vinorelbine ( $p=0.021)$, however Multivariate Cox regression analysis revealed that combined use with docetaxel was the only independent risk factor for capecitabine-induced PPE $(P=0.001)$. These results are in agreement with the pathogenic bases in terms of toxicity, superimposed by the other molecules associated with capecitabine.

\section{Comorbidities}

The choice of comorbidities studied in our series was based on two approaches; the first one is epidemiological and topographic, based on the frequency of these comorbidities in Morocco, particularly, dermatophytosis and onychomycosis [21], micro and macroangiopathic complications and trophic disorders associated with diabetes, with distal topography. The second approach is histological and pathogenic, indeed most clinicians 
believe that HFS is a type of inflammation mediated by COX-2 over-expression in the palm and plantar which may be triggered directly or indirectly by capecitabine or its metabolites [22], histological examination of the tissues affected by PPE, revealed systemic inflammatory changes [23], dermal changes that includes dilated blood vessels, papillary edema, and a sparse superficial perivascular lymphohistiocytic infiltrate [8], in addition to this inflammatory theory, an added vascular component remains very likely, in particular given the localization at the extremities and the role of capillary microtrauma $[24,25]$. So possible implication of diabetes and/ or chronic venous insufficiency and/or eczema and/ or dermatophytosis and onychomycosis as risk factors could involve a micro and macroangiopathic vascular component in the pathogenesis of EPP, and reinforce theories on the involvement of inflammatory phenomena [22] or even the interest of a microbial pro-inflammatory component.

Among the studied comorbidities, those which were significant are, diabetes $(p=0.03)$ and the combination of two or more factors ( $p=0.02$ ), which could possibly imply the interest of a macro and microangiopathic component. This will require a qualitative biologic and radiological exploration to objectify the importance of this component (diabetes, $p=0.03$ ) in other studies, or even new models of inflammatory and vascular synergy in PPE (association of two factors or more, $p=0.02$ ).

Other factors have been identified in the literature, notably the previous occurrence of chemotherapy-related stomatitis before the development of PPE, which was associated with the appearance of PPE $(P=0.029)$ [15], a continuous pattern and/or total cumulative dose of capecitabine $[7,14]$.

The results of this study are limited by inhomogeneous sampling with a high prevalence of female (70\%) and breast cancer (62\%), as well as by the need for better paraclinical exploration of the comorbidities.

In our cohort, and in comparison, with the data in the literature $[4,7,15,26]$ : The risk factor identified in accordance with the data in the literature remains, combined treatment $(p=0.01)$. Risk factors identified in opposition to the data in the literature were sex $(p=0.04)$ and primary breast neoplasia $(p=0.03)$. Comorbidities requiring new models of pathogenic apprehension and other more specific studies, diabetes $(p=0.03)$ and the combination of two or more of studied factors $(p=0.02)$.

\section{Conclusion}

This clinical study provides an epidemiological mapping of PPE in cancer patients on capecitabine treatment and theorizes new questions, based on a study of risk factors, on new models of synergy, notably inflammatory and vascular, in the etiopathogen- esis of PPE.

\section{Conflicts of Interest}

None.

\section{References}

1. Zuehlke RL (1974) Erythematous eruption of the palms and soles associated with mitotane therapy. Dermatologica 148: $90-92$.

2. Brad R Baack, WHC Burgdorf (1991) Chemotherapy-induced acral erythema. Journal of the American Academy of Dermatology 24: 457-461.

3. Nikolaou V, Syrigos K, Saif MW (2016) Incidence and implications of chemotherapy related hand-foot syndrome. Expert Opinion on Drug Safety.

4. Gressett SM, Stanford BL, Hardwicke F (2006) Management of hand-foot syndrome induced by capecitabine. J Oncol Pharm Pract 12: 131-141.

5. Lacouture ME, Wu S, Robert C, Atkins MB, Kong HH (2008) Evolving strategies for the management of hand-foot skin reaction associated with the multitargeted kinase inhibitors sorafenib and sunitinib. Oncologist 13: 1001-1011.

6. Feliu J, Escudero P, Llosa F, Bolaños M, Vicent JM, et al. (2005) Capecitabine as first-line treatment for patients older than 70 years with metastatic colorectal cancer: An Oncopaz Cooperative Group study. J Clin Oncol 23: 3104-3111.

7. Abushullaih S, Saad ED, Munsell, Hoff PM (2002) Incidence and severity of hand-foot syndrome in colorectal cancer patients treated with capecitabine: a single-institution experience. Cancer Invest 20: 3-10.

8. Nagore E, Insa A, Sanmartin O (2000) Antineoplastic therapyinduced palmar-plantar erythrodysesthesia (hand-foot) syndrome. Incidence, recognition and management. Am J Clin Dermatol 1: 225-234.

9. Gómez-Martin C, Sánchez A, Irigoyen A, Llorente B, Pérez $B$, et al. (2012) Incidence of hand-foot syndrome with capecitabine in combination with chemotherapy as firstline treatment in patients with advanced and/or metastatic gastric cancer suitable for treatment with a fluoropyrimidine-based regimen. Clin Transl Oncol 14: 689-697.

10. Yucel I, Guzin G (2008) Topical henna for capecitabine induced hand-foot syndrome. Invest New Drugs 26: 189-192.

11. Murugan K, Ostwal V, Carvalho MD, D'souza A, Achrekar MS, et al. (2016) Self-identification and management of hand-foot syndrome (HFS): effect of a structured teaching program on patients receiving capecitabine-based chemotherapy for colon cancer. Support Care Cancer 24 : 25752581.

12. El-Helw L, Coleman RE (2005) Reduced dose capecitabine is an effective and well-tolerated treatment in patients with metastatic breast cancer. Breast 14: 36874 .

13. O'Shaughnessy J, Blum J (2000) A retrospective evaluation of the impact of dose reduction in patients treated with Xeloda (capecitabine). Proc Am Soc Clin Oncol 19: 104A.

14. Lévy E, Piedbois $P$, Buyse $M$, Pignon JP, Rougier $P$, et al. (1998) Meta-AnalysisGroup in Cancer. Toxicity of fluorouracil in patients with advanced colorectal cancer: Effect of administration schedule and prognostic factors. J Clin Oncol 16: 3537-3541.

15. Heo YS, Chang HM, Kim TW, Ryu MH, Ahn JH, et al. (2004) Hand-Foot Syndrome in Patients Treated with 
Capecitabine-Containing Combination Chemotherapy. J Clin Pharmacol 44: 1166.

16. Suzuki S, Nawata S, Inada Y, Sato D, Kusano J, et al. (2018) A cross-sectional survey of methods for controlling hand-foot syndrome in patients receiving capecitabine treatment. Mol Clin Oncol 9: 443-448.

17. Hoff PM, Ansari R, Batist G, Cox J, Kocha W, et al. (2001) Comparison of oral capecitabine versus intravenous fluorouracil plus leucovorin as first-line treatment in 605 patients with metastatic colorectal cancer: results of a randomized Phase III study. J Clin Oncol 19: 2282-2292.

18. Van Cutsem E, Twelves C, Cassidy J, Allman D, Bajetta E, et al. (2001) Oral capecitabine compared with intravenous fluorouracil plus leucovorin in patients with metastatic colorectal cancer: Results of a large Phase III study. J Clin Oncol 19: 4097-4106.

19. Reichardt P, von Minckwitz G, Thuss-Patience PC, Jonat W, Kölbl H, et al. (2003) Multicenter phase II study of oral capecitabine (Xeloda) in patients with metastatic breast cancer relapsing after treatment with a taxane-containing therapy. Ann Oncol 14: 1227-1233.

20. Miller KD, Chap LI, Holmes FA, Cobleigh MA, Marcom PK, et al. (2005) Randomized phase III trial of capecitabine compared with bevacizumab plus capecitabine in patients with previously treated metastatic breast cancer. J Clin Oncol 23: 792-799.

21. Halim I, El Kadioui F, Soussi Abdallaoui M (2013) Onychomycosis in Casablanca (Morocco). J Mycol Med 23: 9-14.

22. Lou Y, Wang Q, Zheng J, Hu H, Liu L, et al. (2016) Possible Pathways of Capecitabine-Induced Hand-Foot Syndrome. Toxicol 29: 1591-1601.

23. Lassere Y, Hoff P (2004) Managment of hand foot syndrome in patient treated with capecitabine (Xeloda). Eur $\mathrm{J}$ Oncol Nurs 1: S31-S40.

24. Saif MW, Elfiky A, Diasio R (2000) Hand-foot syndrome variant in a dihydropyrimidine dehydrogenase-deficient patient treated with capecitabine. Clin Colorectal Cancer 6: 219-223.

25. Lyass O, Uziely B, Ben-Yosef R, Tzemach D, Heshing NI, et al. (2000) Correlation of toxicity with pharmacokinetics of pegylated liposomal doxorubicin (Doxil) in metastatic breast carcinoma. Cancer 89: 1037-1047.

26. Yap YS, Kwok LL, Syn N, Chay WY, Chia JWK, et al. (2017) Predictors of Hand-Foot Syndrome and Pyridoxine for Prevention of Capecitabine-Induced Hand-Foot Syndrome A Randomized Clinical Trial. JAMA Oncol 3 : 1538-1545. 